Ramírez Suárez, Á. (2013). Análisis de eficiencia económica de fincas arroceras: una aplicación de una función determinística de ingresos brutos frontera. Revista Lebret (5). Bucaramanga, Colombia: Universidad Santo Tomás, pp 213-240

\title{
Análisis de eficiencia económica de fincas arroceras: una aplicación de una función determinística de ingresos brutos frontera*
}

\section{Analysis of Economic Efficiency of Rice Farms: An Application of a Deterministic Gross Revenue Frontier Function Approach}

\section{Resumen}

Álvaro Ramírez Suárez ${ }^{1}$

El análisis sobre la eficiencia económica de la producción de arroz riego a nivel de fincas, se centró en medir indicadores de eficiencia técnica, distributiva y económica usando una muestra de corte transversal, empleando el enfoque de funciones frontera determinísticas mediante la especificación y ajuste de una función de ingresos brutos. Los resultados empíricos soportan que, un agricultor promedio podría aumentar significativamente los rendimientos por hectárea hasta en un $53 \%$, reducir los costos variables unitarios hasta en un $34.5 \%$ y aumentar las ganancias brutas antes de cubrir los costos fijos hasta en un $70 \%$ si usara y combinara los recursos de tierra, mano de obra e insumos al nivel de los agricultores más eficientes económicamente en la región. Al final, se plantean sugerencias con el objeto de cerrar brechas de rendimiento.

\section{Palabras clave}

Arroz de Riego, Eficiencia Productiva, Eficiencia Económica, Eficiencia Distributiva, Eficiencia Técnica

\section{Código de Clasificación JEL: Q12}

\begin{abstract}
This paper aimed at ascertaining the level of technical, distributive and economic efficiency through cross sectional data from rice farmers's fields. With this end in mind, a deterministic gross revenue frontier function approach was used. The empirical evidence supported the notion that an average rice farmer in this region could increase current rice yields up to $53 \%$, reduce unit variable costs up to $34.5 \%$ and increase gross revenue (before covering fixed costs) up to $70 \%$ if $\mathrm{He} / \mathrm{sh}$ were to use and combine land, labor and purchased inputs at similar levels and fashions as those employed by bestpractice farmers in the sample. At the end suggestions are raised in order to bridgeyield gaps.
\end{abstract}

\section{Keywords}

Irrigated Rice, Productive Efficiency, Economic Efficiency, Distributive Efficiency, Technical Efficiency.

\footnotetext{
* Se agradece a la Federación Nacional de Arroceros de Colombia por facilitar los datos de campo usados en este estudio mientras el autor era investigador del Fondo Latinoamericano de Arroz Riego FLAR en el Centro Internacional de Agricultura Tropical CIAT. Los resultados, conclusiones, errores y omisiones son responsabilidad exclusiva del autor y no comprometen a las entidades mencionadas.

1 Ph. D. en Economía Agrícola, Universidad del Estado de Oklahoma. Docente de las Facultades de Administración de Empresas Agropecuarias y Economía. Universidad Santo Tomás, Bucaramanga, Colombia. Correo electrónico: arscol45@yahoo.com
} 


\section{Introducción}

Los cultivadores de arroz en Colombia se enfrentan al reto de competir con las crecientes importaciones de arroz, producto de la desgravación gradual de la protección arancelaria resultado de los acuerdos y tratados de libre comercio. Para ello, los agricultores necesitan producir arroz a un costo promedio inferior a US\$ 150 tonelada en finca. Durante la última década, el rendimiento promedio nacional de arroz paddy verde ha variado en el rango de 5.0 a $6.5 \mathrm{t} \mathrm{ha}^{-1}$ (DANE y FEDEARROZ, 2011). Este nivel de productividad es inferior al rendimiento alcanzado por los mejores productores $\left(9\right.$ a $\left.11 \mathrm{t} \mathrm{ha}^{-1}\right)$ con la tecnología disponible. En consecuencia, el costo unitario promedio nacional a precios del 2013 se mantiene en un nivel mayor a US\$ 250 por tonelada (FEDESARROLLO, IQUARTIL, y MADR, 2012). Este costo es posible reducirlo a la mitad o más con la asistencia de programas enfocados a cerrar las brechas actuales de productividad entre agricultores como es el propósito central del programa de adopción masiva de tecnología AMTEC desarrollado por la Federación Nacional de Arroceros (FEDEARROZ, 2012). Lo anterior bajo la hipótesis de que la eficiencia técnica, distributiva y económica de la producción actual de arroz en las fincas se puede aumentar si los agricultores hacen una mejor asignación y un uso más eficiente de los recursos productivos con la tecnología existente.

Los estudios publicados sobre la eficiencia productiva del arroz a nivel de fincas usando enfoques paramétricos como las funciones de producción frontera en Colombia son inexistentes. Ramírez (1986) y Sanint (1994) midieron la eficiencia económica de la producción de arroz riego mediante funciones frontera estocásticas pero los trabajos no fueron publicados en revistas indexadas. A nivel mundial, la tendencia en la mayoría de estudios de eficiencia en arroz ha sido a medir la eficiencia técnica usando funciones fronteras determinísticas y estocásticas estimadas con datos de corte transversal, y muy pocos a estudiar la eficiencia distributiva y económica o a emplear datos de corte longitudinal o datos de panel (Idiong, 2007; Shehu, Mshelia \& Tashikalma, 2007; Nguyen, Kawaguchi, \& Suzuki, 2003; Xu \& Jeffrey, 1998; Audibert, 1997; Tadesse \& Krishnamoorty, 1997; Trewin, Weiguo, \& Erwidodo, 1995; Batesse \& Coelli, 1992; Dawson \& Lingard, 1991; Ali \& Flinn, 1989; Dawson, 1989; Flinn \& Ali, 1986; Lingard, Castillo, y Jayasuriya, 1983).

Recientemente, la literatura sobre eficiencia económica en fincas arroceras se viene aumentando con algunos análisis frontera no paramétricos de eficiencia técnica y de escala basados en programación lineal tipo DEA ${ }^{2}$ (Gee-Thean, Ismail \& Harron, 2012; Dhungona, Nuthall \& Nartea , 2004; Wadud \& White, 2000). Asimismo, son muy escasos los estudios de eficiencia en arroz enfocados a determinar los factores causales y a explicar las fuentes de dichas ineficiencias (Islam, Sumelius \& Backman, 2012; Liu \& Zhuang, 2000; Tian \& Wan, 2000).

2 Siglas de la técnica conocida como: Data Envelopment Analysis. 
El objetivo de este análisis es, por tanto, estimar indicadores de eficiencia técnica, distributiva y económica de la producción de arroz para la región central arrocera de Colombia usando una función de producción frontera determinística. Esto con el fin de contribuir a esclarecer si para aumentar la productividad promedia, reducir el costo unitario y aumentar la competitividad del cultivo del arroz, es suficiente mejorar la eficiencia técnica y distributiva de la producción o, en cambio, se necesita desarrollar nuevas tecnologías. En el primer caso, este tipo de información básica debe servir de línea base al sector arrocero en el diseño y ejecución de líneas de acción de investigación adaptativa, capacitación y de transferencia de tecnología a los agricultores, enfocadas a aumentar los rendimientos actuales y reducir los costos de producción usando la oferta tecnológica actual.

La primera sección del artículo revisa los conceptos teóricos de eficiencia y de funciones frontera. En la segunda sección se exponen los principales métodos para medir eficiencia a partir de la estimación de funciones frontera. En la tercera sección se discuten los datos y el modelo empírico usado para estimar una función de ingresos brutos frontera de tipo determinístico a partir del cual se derivaron indicadores de eficiencia técnica, distributiva y económica en arroz riego. En la penúltima sección se analizan los resultados y en la última, se discuten las conclusiones e implicaciones del estudio.

\section{Marco Teórico}

\subsection{Concepto de función frontera}

La teoría económica de la producción está construida sobre principios de racionalidad en el comportamiento de los agentes económicos, quienes intentan maximizar el valor de la producción o minimizar el costo de las actividades de producción de bienes y servicios con el fin de maximizar las ganancias de su actividad económica (Heady, 1952).

Según Heady \& Dillon (1961), la teoría de la producción de la empresa modela dicho comportamiento mediante diferentes relaciones y funciones. La función de producción, por ejemplo, relaciona el máximo producto que puede ser producido de una cantidad dada de recursos dada la tecnología. A su vez, la función de costos expresa el mínimo costo de producir un nivel dado de producto, otorgados los precios de los recursos, del producto y la tecnología. De igual forma, la función de ganancias representa el máximo nivel de ganancias que puede ser logrado dados los precios del producto, los recursos y la tecnología.

El término frontera en estas funciones encierra los conceptos de máximos y mínimos, porque estas fijan límites superiores o inferiores al rango de posibles observaciones en una muestra estadística o en la población de las unidades productivas o empresas. Las cantidades por las cuales una empresa se encuentra por debajo de 
su función de producción y ganancias frontera, o la cantidad por la cual la empresa se localiza por encima de la función de costos pueden relacionarse como medidas de ineficiencia. Según Dillon (1968), en el mundo real una firma puede no alcanzar el objetivo de maximizar ganancias debido a: (1) ineficiencias técnicas, distributivas y de escala sobre las cuales tiene alguna forma de control; (2) factores aleatorios fuera del control de la empresa y errores de medición.

La medición de ineficiencias en los procesos productivos ha sido la principal motivación para el estudio de funciones fronteras. La estimación de funciones frontera implica que la variable dependiente constituya un límite envolvente de las observaciones que dan origen a la función (Greene, 1980). La función frontera no presupone eficiencia técnica, y en este sentido se diferencia de las funciones econométricas promedias ${ }^{3}$ de producción, costos y ganancias las cuales asumen la existencia de eficiencia técnica y el uso de productos e insumos homogéneos.

\subsection{Concepto de Eficiencia}

Según la teoría neoclásica de la firma, el objetivo de la empresa es maximizar la ganancia financiera, sujeta a los niveles y precios de mercado de los recursos y productos y la función de producción. Bajo esta teoría se asume que la empresa opera de una manera técnicamente eficiente. Por tanto, el mayor interés analítico de la teoría es conceptualizar las ineficiencias distributivas y económicas, y derivarlas de la función de producción. No obstante, numerosos estudios empíricos documentan que las ineficiencias técnicas entre unidades productivas son sustanciales y significativas (Minh \& Long, 2009; Nyemeck, Sylla, Diarra, \& Nyambi, 2003; Bravo-Ureta \& Pinheiro, 1993; Dawson, Lingard, \& Woodford, 1991; Batesse \& Coelli, 1988; Ramírez, 1986; Flinn \& Ali, 1986; Kalijaran \& Flinn,1983). La existencia de ineficiencias técnicas, distributivas y económicas ofrecen una oportunidad para incrementar la producción de la empresa con los recursos existentes (sin recursos adicionales), si los factores que causan las ineficiencias pueden ser identificados.

Matemáticamente, la eficiencia en la producción se deriva de las siguientes funciones:

- la función de producción: $\mathrm{y}=\mathrm{f}(\mathrm{x})$ la cual muestra la máxima producción posible de obtener de un vector dado de insumos (x).

- la función de costos: $\mathrm{c}=\mathrm{c}(\mathrm{w}, \mathrm{y})$ la cual muestra el costo mínimo necesario para producir (y) a los precios de los insumos (w).

- la función de ganancias: $\pi=\pi(\mathrm{p}, \mathrm{w})$ la cual muestra la máxima ganancia disponible al precio del producto (p) y de los insumos (w). 
Donde:

$\begin{array}{lll}\mathrm{f}, \mathrm{c}, \pi & = & \text { Funciones diferenciables } \\ \mathrm{x} & = & \text { Cantidad de recursos disponibles } \\ \mathrm{y} & = & \text { Cantidad de producto posible de obtener } \\ \mathrm{p} & = & \text { Precio de mercado del producto } \\ \mathrm{w} & = & \text { Precio de mercado de los insumos }\end{array}$

La ineficiencia técnica resulta de cierta inhabilidad del productor para lograr el máximo producto de los recursos usados, es decir, de su falta de capacidad para usar la tecnología disponible eficientemente y emplear los recursos en la cantidad y combinación necesarios para lograr el máximo producto. A su vez, la ineficiencia distributiva o de precios resulta de la inhabilidad del empresario para usar la cantidad de recursos óptima o de mínimo costo para obtener un nivel dado de producto y maximizar las ganancias, dadas las señales de precios del producto y precios de los recursos en el mercado. La ineficiencia económica es por tanto, la resultante de la existencia de ineficiencias técnicas y distributivas.

\section{Funciones de producción frontera y la medición de eficiencia}

\subsection{El enfoque de Farrell}

El concepto de eficiencia económica constituye el fundamento teórico para la medición del desempeño relativo de las empresas. El trabajo pionero de Farrell (1957) definió los conceptos de eficiencia técnica, distributiva y económica a nivel de la firma y de la industria y trazó el marco conceptual para su medición empírica mediante funciones frontera no paramétricas y paramétricas (Greene, 1980). Farell definió la frontera de producción eficiente como el locus de niveles de producción máximos posibles de alcanzar dados los niveles de recursos y la tecnología. Asumiendo un ambiente determinístico, mercados competitivos para los recursos y retornos constantes a escala ${ }^{4}$, inicialmente derivó una isocuanta unitaria de producción no paramétrica (en el espacio de recursos o mapa de isocuantas) para calcular los índices de eficiencia técnica y distributiva individual de la empresa.

La Figura 1 ilustra el concepto de eficiencia de Farrell (1957). Se asume que el plan de producción de la empresa es producir un solo producto Y, mediante el

4 Retornos a escala: un incremento proporcional en el uso de todos los recursos produce un incremento proporcional en la cantidad o retorno del producto. 
empleo de dos insumos $\left(\mathrm{X}_{1} \mathrm{y}_{2}\right)$. La función de producción frontera de la firma es, por tanto, $\mathrm{Y}^{0}=\mathrm{f}\left(\mathrm{X}_{1}, \mathrm{X}_{2}\right)$ la cual se caracteriza por retornos constantes a escala. Bajo este supuesto, la isocuanta $\mathrm{Y}^{0}$ es una isocuanta unitaria y representa las distintas combinaciones de $X_{1}$ y $X_{2}$ que la firma debe usar para ser técnicamente eficiente en la producción de $\mathrm{Y}^{0}$. Por definición, la firma no puede producir por debajo de la isocuanta $\mathrm{Y}^{0}$. Estos puntos no son factibles. Si la firma produce por encima de la isocuanta $\mathrm{Y}^{0}$, como es el caso del punto A en la Figura 1, es técnicamente ineficiente.

Asúmase que la firma actualmente produce en el punto A. En este punto, la empresa usa $\mathrm{X}_{1}^{0}, \mathrm{X}_{2}^{0}$ para producir $\mathrm{y}^{0}$. Este punto está por encima de la isocuanta $\mathrm{Y}^{0}$ lo cual es técnicamente ineficiente porque $\mathrm{Y}^{0}>\mathrm{y}^{0}$. Para ser técnicamente eficientemente con ese nivel y combinación de insumos $\left(\mathrm{X}_{1}^{0}, \mathrm{X}_{2}^{0}\right)$, la empresa debe producir la cantidad $\mathrm{Y}^{0}$ en el punto $\mathrm{B}$. La relación $\mathrm{OB} / \mathrm{OA}$ mide la eficiencia técnica y corresponde a la relación entre la cantidad de insumos para producir $\mathrm{y}^{0}$ de manera técnicamente eficiente sobre la isocuanta $\mathrm{Y}^{0}(\mathrm{OB})$, y la cantidad realmente usada por la firma (OA).

La línea PP es la línea isocosto y representa la relación de precios de los insumos o línea de presupuesto. En el punto A la firma es también distributivamente ineficiente, porque el costo de producir $\mathrm{y}^{0}$ en el punto $\mathrm{A}$ es mayor que el costo de producir $\mathrm{Y}^{0}$ en el punto $\mathrm{C}$ el cual corresponde al costo mínimo de producir $\mathrm{y}^{0} \mathrm{de}$ manera técnicamente eficiente. Sin embargo, solo en el punto D la firma es técnica y distributivamente eficiente para producir $\mathrm{Y}^{0}$ porque en este punto la tasa marginal de sustitución de los insumos es igual a la relación de sus precios y la firma en este punto minimiza el costo y maximiza la ganancia en la producción de $\mathrm{Y}^{0}$. La relación $\mathrm{OD} / \mathrm{OB}$ mide la eficiencia distributiva la cual corresponde a la relación entre el costo mínimo para producir $\mathrm{Y}^{0}$ de manera técnica y distributivamente eficiente (OD) ${ }^{5}$ y el costo técnicamente eficiente de producir $\mathrm{Y}^{0}$ en el punto A $(\mathrm{OB})$. Por definición, todos los puntos a lo largo de la isoclina OA tienen la misma eficiencia distributiva. Bajo el supuesto de retornos constantes a escala la eficiencia económica (EE) de la firma es igual al producto de la eficiencia técnica (ET) y distributiva (ED).

Esto es, $\mathrm{EE}=\mathrm{OB} / \mathrm{OA} * \mathrm{OD} / \mathrm{OB}=\mathrm{OD} / \mathrm{OA}$. Es decir, que si la firma es eficiente técnica y distributivamente su costo de producir $\mathrm{Y}^{0}$ es sólo una fracción (OD/OA) del costo actual de producir $\mathrm{y}^{0}$ en el punto A.

$5 \quad$ El costo en el punto D es igual al costo en el punto C porque se ubica a lo largo de la línea isocosto (PP) de la empresa. 
Figura 1. Modelo de eficiencia económica de Farrell

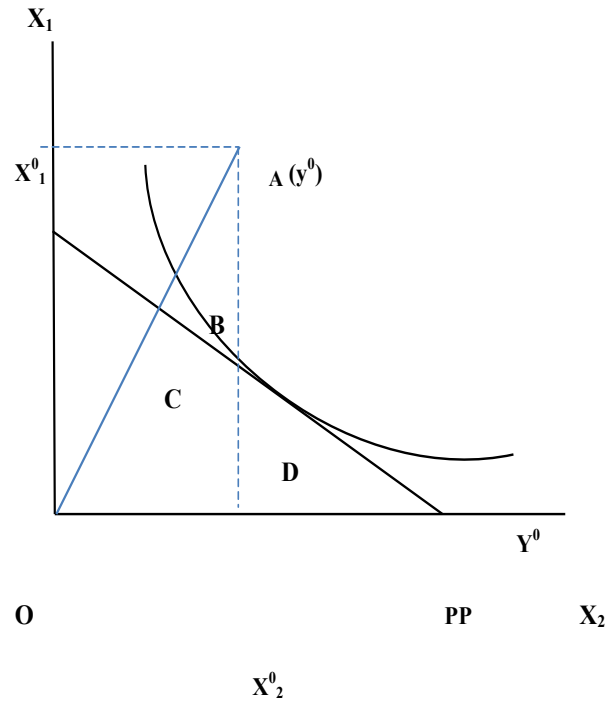

Fuente: Autor

\subsection{Modelos de funciones frontera}

Los modelos de fronteras desarrollados después del trabajo de Farrell (1957) son numerosos. Según Greene (1993) estos modelos se clasifican en dos grupos: paramétricos y no paramétricos. Los modelos paramétricos estiman la frontera eficiente econométricamente asumiendo una forma algebraica funcional específica para la relación $\mathrm{y}=\mathrm{f}(\mathrm{x})$ donde $(\mathrm{y})$ es un vector de producto(s) $\mathrm{y}(\mathrm{x})$ es un vector de recursos productivos. En contraste, los modelos no paramétricos derivan esta frontera empleando métodos de programación matemática (lineal y cuadrática).

A su vez, los modelos paramétricos se distinguen como: determinísticos y estocásticos. Los primeros asumen que todas las desviaciones de la frontera se deben a ineficiencias las cuales son capturadas en el término del error $(\mu)$ de la función $y=f(x)-\mu$. En este caso, se asume que los errores aleatorios y de medición $(v)$ son inexistentes. Los segundos descomponen el término del error ( $(\varepsilon)$ explícitamente en desviaciones debidas a ineficiencia $(\mu)$ y al ruido estadístico clásico (v). Es decir, y = $\mathrm{f}(\mathrm{x})-\varepsilon$. En forma equivalente $\mathrm{y}=\mathrm{f}(\mathrm{x})-(\mu+\mathrm{v})$. Los modelos no paramétricos son determinísticos por definición. 


\subsubsection{Fronteras no-paramétricas.}

El enfoque de Farrell (1957) es no paramétrico, en el sentido de que la isocuanta de producción frontera se construye a partir de una submuestra de las empresas más eficientes dentro de una muestra de empresas usando programación lineal. Además, se asume que la diferencia en producción entre la isocuanta frontera y el valor de una observación dada se debe exclusivamente a ineficiencia técnica (Aigner \& Chu, 1968; Richmond, 1974). Este método presenta la ventaja de que no impone una forma funcional a los datos. Sin embargo, y según Greene (1993), estas presentan dos desventajas: el supuesto de retornos constantes a escala es restrictivo y su extensión a los casos de retornos no constantes o a tecnologías no homotéticas es compleja. Además, el método de derivación de la frontera es sensible a valores extremos de las observaciones y a errores de medición. Asimismo, no se pueden hacer inferencias y pruebas de hipótesis acerca de los estimadores de eficiencia.

Por estas razones, los modelos no paramétricos han tenido pocos seguidores aunque una versión del modelo conocida como Análisis de Datos Envolventes (DEA por sus siglas en inglés) se ha popularizado en los últimos años después del trabajo de Cooper, Seiford \& Tone (2007), y de Liu, Lu, Lu \& Lin (2013). Más recientemente, con la introducción de técnicas estadísticas de bootstrapping las cuales permiten calcular intervalos de confianza para los estimados de eficiencia técnica en los modelos tipo DEA, algunas de las anteriores críticas se han atenuado y su empleo en estudios empíricos se viene acrecentando (Simar \& Wilson, 2000; Thiam, BravoUreta \& Rivas, 2001; Brummer, 2001; Ortner, Hambrusch \& Kirner, 2006; Liu, Lu, Lu \& Lin, 2013).

\subsubsection{Fronteras paramétricas determinísticas.}

Según Greene (1980), Farrell (1957) propuso usar una forma funcional específica (Cobb-Douglas) para estimar la frontera en una forma matemáticamente más simple y al mismo tiempo eliminar las desventajas anteriores. No obstante, Farrell (1957) reconoció que la función Cobb-Douglas limitaba la determinación de la frontera de producción al caso de tecnologías homotéticas y retornos constantes a escala. Aigner \& Chu (1968) propusieron dos métodos para estimar una función de producción frontera del tipo Cobb-Douglas asumiendo un proceso de producción determinístico. En su forma determinística, el modelo Cobb-Douglas y $=\mathrm{f}(\mathrm{x})-\mu$, lo expresaron linealmente como:

$$
\ln y_{j}=\ln \beta_{0}+\sum_{i=1}^{n} \beta_{i} \ln X_{i j}-u_{j}, \quad u_{j} \geq 0
$$

En donde: $\beta_{\mathrm{i}}$ representa los coeficientes estimados de la regresión para cada recurso i usado en el proceso productivo por cada una de las $\mathrm{j}$ empresas en la población. El término del error truncado $\left(u_{j}\right)$ se asume que se distribuye en forma 
idéntica e independiente con media no negativa y varianza conocida. Así, $\mathrm{u}_{\mathrm{j}}$ forza a que: $\ln \mathrm{y} \leq \ln \mathrm{f}(\mathrm{x})$. Es decir, que todas las observaciones caigan arriba o debajo de la función frontera. Aigner \& Chu (1968) estimaron estadísticamente los parámetros $\beta_{\mathrm{i}}$ usando dos métodos econométricos: mínimos cuadrados ordinarios (MCO) y máxima verosimilitud (MVS) haciendo supuestos explícitos sobre la forma de la distribución del término de error (u).

Las estimaciones hechas de este modelo sugirieron que: (1) El método de mínimos cuadrados ordinarios $(\mathrm{MCO})$ rendía estimadores insesgados de todos los parámetros excepto del intercepto; (2) El método de máxima verosimilitud rendía estimadores que no eran consistentes y asintóticamente eficientes, porque en este caso el rango de los valores a ser estimados de la variable dependiente (y) depende de los valores de los parámetros $\left(\beta_{\mathrm{i}}\right)$. Por tanto, la escogencia de la distribución de (u) era crítica, porque distribuciones distintas rinden estimadores diferentes.

Para corregir los problemas de estimación del intercepto, Richmon (1974) y Greene (1980) desarrollaron dos métodos de estimación alternativos conocidos como mínimos cuadrados ordinarios corregidos (MCOC). Richmon (1974) propuso un método de corrección basado en el supuesto de que (u) sigue una distribución truncada tipo gamma forzando al término de error (u) a ser positivo y estimó los parámetros de la función usando mínimos cuadrados ordinarios $\mathrm{MCO}$, llamando a esta técnica mínimos cuadrados corregidos MCCO. Esta técnica también se conoce como mínimos cuadrados ordinarios corregidos por translación de la media (Álvarez, 2001).

A su vez Greene (1980) asumió que la media de (u) es $\bar{\mu}$. Entonces, el modelo (1) puede reescribirse como:

$$
\ln y=\left(\beta_{0}-\bar{\mu}\right)+\sum_{i=1}^{n} \beta_{i} \ln x_{i}-(u-\bar{\mu})
$$

Donde: la media del nuevo término del error $(\mathrm{u}-\bar{\mu})$ es cero $(0)$. En este caso, el error cumple todas las condiciones regulares, excepto el criterio de normalidad. Por tanto, el modelo (3) puede estimarse por MCOC para obtener los mejores estimadores insesgados de $\left(\beta_{0}-\mu\right)$ y de los $\left(\beta_{\mathrm{i}}\right)$. Esta técnica a su vez se conoce como mínimos cuadrados ordinarios corregidos por translación al extremo (Álvarez, 2001).

\subsubsection{Fronteras paramétricas estocásticas.}

Los modelos determinísticos ignoran que la eficiencia de la empresa puede ser afectada por factores exógenos negativos fuera de su control, tales como: clima, dotación de recursos de producción, desempeño de la maquinaria y mano de obra, heterogeneidad entre las empresas entre otros factores. Para corregir este problema (Aigner, Lovell, \& Schmidt, 1977; Meeusen \& Van Den Broeck, 1977) propusieron el 
modelo de fronteras estocásticas. Estos autores formularon el modelo de producción frontera estocástica como:

$$
\begin{aligned}
& y=f(x) e^{\varepsilon} \text { o alternativamente } \\
& y=f(x) e^{v+\mu}
\end{aligned}
$$

Donde: $(\varepsilon)$ es el término del error compuesto, $(v)$ es el término de error simétrico, ( $\mu$ ) es el término de error truncado de ineficiencia técnica con $(\mu \geq 0)$ y (e) es el operador exponencial (número neperiano $e$ ). Estas condiciones hacen que todas las observaciones de las empresas caigan sobre la frontera o por debajo de la misma. La inclusión del término de error simétrico $(v)$ resuelve el problema de violación de la condición de regularidad de los estimadores de máxima verosimilitud. El efecto de la inclusión del término de ineficiencia técnica $(\mu)$ es posicionar cada función de producción individual de la empresa a lo largo o por debajo de la frontera. De esta formulación, se desprende que las funciones de producción "promedia" y determinística están anidadas dentro de la función frontera estocástica (4) anterior. Si $\sigma_{\mu}=0$ la frontera estocástica colapsa en una función "promedia". Si $\sigma_{v}=0$ la frontera estocástica termina en una función determinística. Por lo tanto, a partir de la función estocástica se pueden hacer pruebas de hipótesis para determinar cuál especificación puede ser la más apropiada.

Los trabajos liderados por Greene y los avances en las tecnologías de información, ampliaron las posibilidades teóricas y computacionales para ajustar funciones frontera estocásticas en diferentes sectores productivos de la economía (Greene W. H., 1980; Greene H. W., 1993; Greene W. H., 2002a; Greene W. H., 2002b) El modelo estocástico continúa siendo el más empleado en la literatura para hacer análisis de eficiencia económica de los procesos productivos dada su mayor robustez teórica (Bravo-Ureta \& Pinheiro, 1993; Fried, Lovell, \& Schmidt, 1993; Coelli, Prasada, \& Batesse, 1998; Alvarez, 2001; Thiam, Bravo-Ureta \& Rivas, 2001; Kwon \& Lee, 2004; (Bravo-Ureta, Solis, Moreira, Maxipani, Thiam, \& Rivas, 2007, Minh \& Long, 2009; Galawat \& Yabe, 2012).

\section{Datos y modelo empírico de función frontera determinística}

Para adelantar el presente estudio se usó información de corte transversal compuesta por 86 fincas de arroz de riego mecanizado, localizadas en los Departamentos del Tolima y Huila correspondientes a la región central, considerada la principal zona productora de arroz de Colombia. Los datos provienen de la encuesta nacional arrocera adelantada por la Federación Nacional de Arroceros en el 2005. La región central es relativamente homogénea en términos agroecológicos. Por tanto, los agricultores de arroz en la muestra enfrentan una oferta tecnológica 
relativamente homogénea en función del tipo de variedades a sembrar y las prácticas de manejo agronómico del cultivo para lograr altos rendimientos. Igualmente, los cultivadores enfrentan relaciones de precios relativos del arroz y de los recursos con ligeras variaciones entre fincas. No obstante, la región es bastante heterogénea en función del tipo de productor, el tamaño de los cultivos, las características socio-culturales y demográficas, dotación de recursos humanos y de capital, y capacidad de negociación de los agricultores (Ramírez, 1986). Por tanto, se espera que estas diferencias conduzcan a un uso diferenciado de la tecnología disponible correspondiente con la habilidad de los agricultores para leer adecuadamente las señales de precios de los mercados y aplicar eficientemente la tecnología disponible.

El modelo empírico usado en este estudio para estimar la eficiencia productiva en el cultivo del arroz consiste de una sola ecuación, la cual se especificó como una función de ingresos brutos ${ }^{6}$ frontera determinística, equivalente a una función de producción escalada por el precio del arroz y el precio de los recursos de producción. Siguiendo a Greene (1980) el modelo determinístico se seleccionó por su atractivo de no requerir un supuesto explícito sobre la forma de distribución del término del error truncado $(\mu)$ al poderse estimar usando el método de mínimos cuadrados corregidos MCCO. Este enfoque debe facilitar el proceso de estimación econométrica de los indicadores de eficiencia económica por parte de instituciones, empresas e individuos no especializados en economía y que no tengan acceso a programas computacionales avanzados como: FRONTIER (Coelli, 1996) y LIMDEP (Greene, 1995).

$\mathrm{Al}$ efecto, se ha documentado empíricamente que los indicadores de eficiencia son sensibles a los métodos de estimación usados. Así, los modelos determinísticos generalmente arrojan indicadores de ineficiencia mayores que los derivados de fronteras estocásticas. Esto en parte se explica en el supuesto del modelo de que, las desviaciones de la frontera, se atribuyen sólo a ineficiencias técnicas. En este sentido, los índices de eficiencia constituyen un límite superior al estimado de ineficiencia. Sin embargo, las distribuciones de los índices de ineficiencia son similares y ordenan las observaciones según niveles de eficiencia de manera semejante a los métodos estocásticos y determinísticos no paramétricos (Minh \& Long, 2009; Jaforullah \& Premachandra, 2003; Wadud, 2003; Sharma, Leung \& Zalesqui, 1999; Ahmad \& Bravo-Ureta, 1996; Neff, Garcia \& Nelson, 1993; Bravo-Ureta \& Rieger, 1990).

Para ajustar la función frontera a los datos se seleccionó la forma funcional CobbDouglas, aunque confinar el análisis a esta sola forma funcional puede ser restrictivo (dados los supuestos de retornos constantes a escala y tasas constantes de sustitución de insumos), algunos estudios empíricos realizados en el cultivo del arroz demuestran que la selección de la forma algebraica de la función (ejemplo: polinomial, translog) tiene bajo impacto sobre las medidas de eficiencia (Kwon \& Lee, 2004; Ekanayake \& Jayasuriya, 1987; Dawson, 1985).

6 El ingreso bruto se define como la diferencia entre el ingreso total menos los costos variables. 
El modelo determinístico de la función de ingresos brutos frontera tipo CobbDouglas se especificó de la siguiente forma:

$$
\ln Y_{j}=\beta_{0}+\beta_{1} \ln X_{1 j}+\beta_{2} \ln X_{2 j}+\beta_{3} \ln X_{3 j}+\beta_{4} \ln X_{4 j}+\beta_{5} \ln X_{5 j}-\mu_{j}
$$

Donde:

$\mathrm{Y}_{\mathrm{j}}=$ Ingreso bruto de la $\mathrm{j}_{\text {ésima }}$ finca

$\mathrm{X}_{1}=$ Gasto en semilla de la $\mathrm{j}_{\text {ésima }}$ finca

$\mathrm{X}_{2}=$ Gasto en nitrógeno de la ${ }_{\mathrm{j} \text { ésima }}$ finca

$\mathrm{X}_{3}=$ Gasto en mano de obra de la $\mathrm{j}_{\text {ésima }}$ finca

$\mathrm{X}_{4}=$ Gasto en maquinaria de la $\mathrm{j}_{\text {ésima }}$ finca

$\mathrm{X}_{5}=$ Gasto en tierra de la $\mathrm{j}_{\text {ésima }}$ finca

$\mu_{\mathrm{j}}=$ término del error truncado de la $\mathrm{j}_{\text {ésima }}$ finca.

Los valores de las variables del modelo se estimaron en base a precios constantes del 2013 para el arroz paddy verde y los insumos productivos. La función (5) se estimó usando la técnica de mínimos cuadrados ordinarios corregidos MCOC por extensión del término de error $(\mu)$ al extremo. Esta función se ajustó econométricamente empleando el paquete estadístico IBM-SPSS 20. Los cálculos de las condiciones de primer orden para un óptimo y generar los índices de eficiencia se adelantaron en MS Excel.

Los índices de eficiencia técnica, distributiva y económica por finca y recursos individuales se derivaron de la función de ingresos brutos así:

La eficiencia técnica de la finca $\mathrm{j}_{\text {ésima }}($ ETj) en el uso de todos los recursos se calculó como:

$$
\mathrm{ET}_{\mathrm{j}}=\mathrm{IBO}_{\mathrm{j}} / \mathrm{IBP}_{\mathrm{j}}
$$

Donde:

$\mathrm{IBO}_{\mathrm{j}}$ es el ingreso bruto observado $\left(\mathrm{y}_{\mathrm{j}}\right)$ de la finca $\mathrm{j}_{\text {ésima }}$, e IBP $\mathrm{j}_{\mathrm{j}}$ es el ingreso bruto predicho $\left(\hat{y}_{\mathrm{j}}\right)$ de la finca $\mathrm{j}_{\text {ésima }}$

La eficiencia distributiva de la finca $\mathrm{j}_{\text {ésima }}\left(\mathrm{ED}_{\mathrm{j}}\right)$ en el uso de todos los recursos se derivó como:

$$
\mathrm{ED}_{\mathrm{j}}=\mathrm{IBP}_{\mathrm{j}} / \mathrm{IBOP}_{\mathrm{j}}
$$

Donde: 
IBP $_{j}$ es el ingreso bruto predicho $\left(\hat{y}_{\mathrm{j}}\right)$ de la finca $\mathrm{j}_{\text {ésima }}$ e IBOP ${ }_{\mathrm{j}}$ es el ingreso bruto óptimo de la finca $\mathrm{j}_{\text {ésima }}$ al nivel óptimo de uso de todos los recursos.

La eficiencia económica de la finca $\mathrm{j}_{\text {ésima }}\left(\mathrm{EE}_{\mathrm{j}}\right)$ en el uso de todos los recursos es por tanto:

$$
\mathrm{EE}_{\mathrm{j}}=\mathrm{ET}_{\mathrm{j}} * \mathrm{ED}_{\mathrm{j}}
$$

El nivel óptimo del insumo iésimo en la finca $\mathrm{j}_{\text {ésima }}\left(\mathrm{X}^{*}{ }_{\mathrm{ij}}\right)$ se calculó a partir de las condiciones de primer orden para un óptimo, las cuales se obtuvieron de las derivadas parciales de la función de ingresos brutos (5). Estas expresiones permiten establecer el nivel óptimo de cada recurso el cual se determina donde el valor del producto marginal del $i_{\text {ésimo }}$ recurso es igual al precio del $i_{\text {ésimo }}$ recurso $\left(\mathrm{w}_{\mathrm{i}}\right)$.

Así, la eficiencia distributiva de la $\mathrm{j}_{\text {ésima }}$ finca en el uso del $\mathrm{i}_{\text {ésimo }}$ recurso $\mathrm{ED}_{\mathrm{ij}}$ es:

$$
\mathrm{ED}_{\mathrm{ij}}=\mathrm{IBOP}_{\mathrm{ij}} / \mathrm{IBOP}_{\mathrm{j}}
$$

Donde:

IBOP $_{\text {ij }}$ es el ingreso bruto de la finca $j_{\text {ésima }}$ al nivel óptimo de uso del $i_{\text {ésimo }}$ recurso con todos los demás recursos constantes al nivel usado por la $j_{\text {ésima }}$ finca.

\section{Análisis de resultados}

\subsection{Estimación de la función frontera determinística}

La Tabla 1 reporta los estadísticos descriptivos para los coeficientes técnicos de las variables del modelo (5) y los estimados por mínimos cuadrados ordinarios corregidos MCOC de los coeficientes de la función frontera determinística.

Se observa que, el tamaño promedio de los cultivos de arroz corresponde a pequeñas unidades productivas (7,5 ha con un coeficiente de variación del $85 \%$ ). En general, se estima que los niveles promedios de uso de semillas, nitrógeno, maquinaria y mano de obra, exceden las cantidades recomendadas por los técnicos en esta región. Este resultado confirma las conclusiones de otros estudios que documentan la tendencia de los agricultores en esta región a usar altos niveles de insumos (Ramírez \& Sanint, 1994).

El modelo estimado es altamente significativo como lo indica el valor del estadístico F. Todos los estimados de elasticidades parciales presentan una alta significancia estadística $(\mathrm{P}<0,01)$ para explicar la variabilidad en los ingresos brutos en la producción de arroz y calcular los índices de ineficiencias para las fincas de la muestra en la producción de arroz. La naturaleza estructural de los estimadores de MCOC no evidencia multicolinearidad, un problema estadístico común en la 
estimación de funciones frontera usando datos de corte transversal. Los signos de los coeficientes de regresión son los esperados. El coeficiente de determinación indica que los gastos en semilla, nitrógeno, maquinaria, mano de obra y tierra explican el $99.8 \%$ de la variabilidad en el ingreso bruto observado.

El valor del coeficiente de la función $(0,994)$ confirma la existencia de retornos constantes a escala un supuesto implícito en los modelos econométricos estimados usando modelos de producción tipo Cobb-Douglas. Dadas las elasticidades de producción calculadas, se puede inferir que un cambio porcentual del $10 \%$ en el área sembrada, mano de obra, semilla, nitrógeno y maquinaria, causaría un cambio proporcional del $3,8 \%, 2,3 \%, 1,7 \%, 1,3 \%$ y $1,2 \%$ respectivamente en el ingreso bruto de un agricultor promedio de arroz en la muestra, manteniendo los demás recursos constantes al nivel de uso promedio.

Tabla 1. Estadísticos descriptivos y coeficientes estimados de la función frontera determinística de ingresos brutos, tipo Cobb - Douglas estimada por Mínimos Cuadrados Ordinarios Corregidos MCOC. $\quad(n=85$ observaciones

\begin{tabular}{|c|c|c|}
\hline Variable & Coeficiente técnico por ha & Coeficiente regresión \\
\hline Intercepto & & $\begin{array}{l}2,061 * * * \\
(0,056)\end{array}$ \\
\hline Semilla (kg) & $\begin{array}{l}264,4 \\
(47,6)\end{array}$ & $\begin{array}{l}0,154 * * * \\
(0,017)\end{array}$ \\
\hline Nitrógeno (kg) & $\begin{array}{l}180.4 \\
(52.1)\end{array}$ & $\begin{array}{l}0,135^{* * *} \\
(0,012)\end{array}$ \\
\hline Mano de obra (hr) & $\begin{array}{l}159.6 \\
(87,7)\end{array}$ & $\begin{array}{l}0,239 * * * \\
(0,013)\end{array}$ \\
\hline Maquinaria (hr) & $\begin{array}{l}12,8 \\
(3,5)\end{array}$ & $\begin{array}{l}0,085^{* * *} \\
(0,010)\end{array}$ \\
\hline Tierra (ha) & $\begin{array}{l}7,5 \\
(6,4)\end{array}$ & $\begin{array}{l}0,381 * * * \\
(0,020)\end{array}$ \\
\hline Rendimiento observado ( $\mathrm{Kg}$ de paddy verde) & $\begin{array}{l}6.419 \\
(906,7)\end{array}$ & \\
\hline Ingreso bruto observado (\$) & $\begin{array}{l}1.521 .164 \\
(525.884)\end{array}$ & \\
\hline Coeficiente función & 0,994 & \\
\hline Valor F & $20.299,6^{* * *}$ & \\
\hline R2 ajustado & 99.8 & \\
\hline
\end{tabular}

*** $\mathrm{P}<0,01$. Nota: Los valores entre paréntesis corresponden a los errores estándar de los coeficientes de regresión y las desviaciones estándar para los promedios de los coeficientes técnicos. 


\subsection{Eficiencia técnica, distributiva y económica a nivel de fincas}

La Figura 2 ilustra el histograma de los índices de eficiencia técnica, distributiva y económica calculados para las 85 fincas en la muestra.

Figura 2. Histograma de los índices de eficiencia técnica, distributiva y económica en la producción de arroz de la región central de Colombia
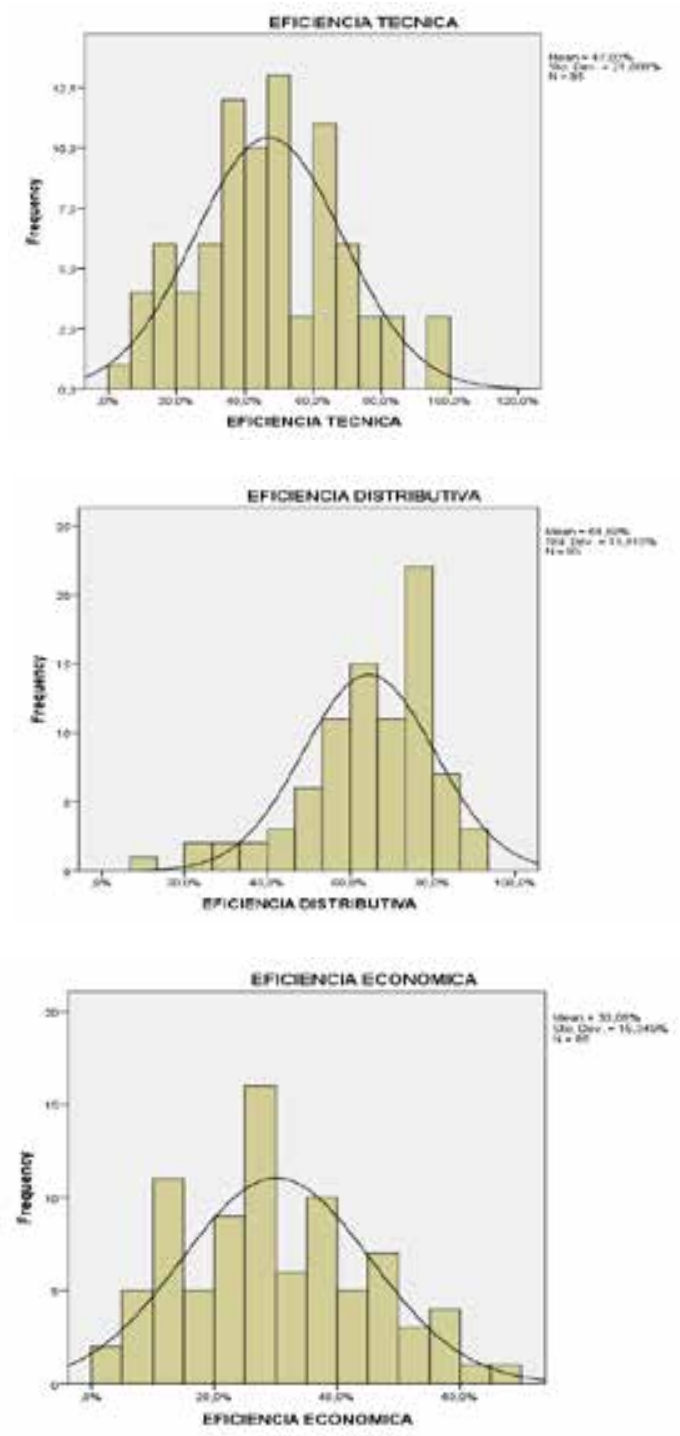

Fuente: Cálculos propios basados en datos de FEDEARROZ 
Los índices de eficiencia variaron ampliamente en el rango del $2.8 \%$ al $100 \%$ para la eficiencia técnica (promedio del $47.0 \%$ ); $8.3 \%$ al $92.4 \%$ para la eficiencia distributiva (promedio del 64.6\%), y del 2.0\% al 68.4\% para la eficiencia económica (promedio del 30.1\%). Estos resultados sugieren que en la región arrocera central de Colombia existía un alto grado de ineficiencias en la producción de arroz en relación a las fincas más eficientes. Cerca de dos tercios de las fincas operaban a un nivel de eficiencia igual o inferior a los promedios de eficiencia técnica, distributiva y económica. Esto indica que estas unidades tenían un nivel de productividad inferior y estaban usando niveles mayores y combinaciones de los recursos productivos más costosas que las de los agricultores ubicados cerca o en la frontera eficiente.

La Tabla 2 muestra la distribución de frecuencias para los índices de eficiencia técnica, distributiva y económica calculados a nivel de las fincas arroceras en la muestra. Asimismo, lista el promedio de los índices de eficiencia para la muestra, la desviación estándar y los valores mínimos y máximos de cada uno de estos indicadores de eficiencia.

Tabla 2. Distribución del número de fincas arroceras según niveles de eficiencia técnica, distributiva y económica en la región central de Colombia $(n=85)$

\begin{tabular}{|c|c|c|c|c|c|c|}
\hline \multirow{2}{*}{$\begin{array}{c}\text { Rango del } \\
\text { índice de } \\
\text { Eficiencia }(\%)\end{array}$} & \multicolumn{2}{|c|}{ Eficiencia técnica } & \multicolumn{2}{|c|}{ Eficiencia distributiva } & \multicolumn{2}{|c|}{ Eficiencia económica } \\
\hline & $\begin{array}{c}\text { Número } \\
\text { fincas }\end{array}$ & $\begin{array}{c}\text { Porcentaje } \\
\%\end{array}$ & $\begin{array}{l}\text { Número } \\
\text { fincas }\end{array}$ & $\begin{array}{c}\text { Porcentaje } \\
\%\end{array}$ & $\begin{array}{l}\text { Número } \\
\text { fincas }\end{array}$ & $\begin{array}{c}\text { Porcentaje } \\
\%\end{array}$ \\
\hline 3 a 13 & 5 & 5.9 & 1 & 1.2 & 12 & 14.1 \\
\hline 13 a 23 & 7 & 8.2 & 2 & 2.4 & 14 & 16.5 \\
\hline 23 a 33 & 8 & 9.4 & 2 & 2.4 & 26 & 30.6 \\
\hline 33 a 43 & 19 & 22.4 & 4 & 4.7 & 16 & 18.8 \\
\hline 43 a 53 & 17 & 20.0 & 6 & 7.1 & 10 & 11.8 \\
\hline 53 a 63 & 6 & 7.1 & 20 & 23.5 & 5 & 5.9 \\
\hline 63 a 73 & 14 & 16.5 & 18 & 21.2 & 2 & 2.4 \\
\hline 73 a 83 & 4 & 4.7 & 28 & 32.9 & 0 & 0.0 \\
\hline 83 a 93 & 2 & 2.4 & 4 & 4.7 & 0 & 0.0 \\
\hline 93 a 100 & 3 & 3.5 & 0 & 0.0 & 0 & 0.0 \\
\hline Promedio & $42.7 \mathrm{a}$ & & $64.6 \mathrm{~b}$ & & $30.1 \mathrm{c}$ & \\
\hline $\begin{array}{l}\text { Desviación } \\
\text { Estándar }\end{array}$ & 20.5 & & 15.9 & & 15.3 & \\
\hline Máximo & 100.0 & & 92.4 & & 68.4 & \\
\hline Mínimo & 2.8 & & 8.3 & & 2.0 & \\
\hline
\end{tabular}

Fuente: Cálculos propios. Las letras (a,b, c) indican que las diferencias en los promedios de eficiencia calculados son estadísticamente significativas con $\mathrm{P}<0.05$ de acuerdo con una prueba t para comparación de medias apareadas. 
Según estos resultados, en términos de eficiencia económica los agricultores podrían aumentar en 70\% o más, los niveles de Ingresos brutos (ganancias brutas antes de cubrir los costos fijos) observados, adoptando y usando la tecnología y los recursos en los niveles y mezclas empleadas por los agricultores más eficientes económicamente, dadas las relaciones de precios del arroz y de los recursos. Específicamente, estos cultivadores podrían reducir los costos unitarios de producción en un $36.4 \%$ o más usando los recursos en forma similar a los agricultores más eficientes distributivamente. Alternativamente, los agricultores menos eficientes técnicamente podrían lograr el mismo nivel de producción observado empleando sólo el 53\% o más de la cantidad actual de recursos de capital y mano de obra, reduciendo así los costos unitarios actuales y aumentando apreciablemente las ganancias. Es decir, existían amplios márgenes para mejorar la productividad y las ganancias del cultivo haciendo una mejor asignación y uso de los recursos con la tecnología disponible.

\subsection{Eficiencia en el uso óptimo de los recursos productivos}

Existen diferentes maneras de identificar las fuentes de posibles ineficiencias en el uso de los recursos productivos (Islam, Sumelius , \& Backman, 2012; Binam, 2004; Ajibefun, Batesse, \& Daramola, 2002; Liu \& Zhuang, 2000; Tian \& Wan, 2000; Admassie, 1999; Coelli \& Batesse, 1996). En este análisis este objetivo se realizó mediante el examen de la eficiencia distributiva de los recursos individuales teniendo en cuenta que este indicador refleja la habilidad de los agricultores para leer los precios de los recursos y el arroz en el mercado y seleccionar la mejor combinación de factores productivos para minimizar el costo unitario y maximizar ganancias. La Figura 3 exhibe el histograma de los índices de eficiencia distributiva para la semilla, nitrógeno, mano de obra, maquinaria y tierra dedicada al cultivo.

Figura 3. Histograma de los índices de eficiencia distributiva en el uso de semillas, nitrógeno, mano de obra, maquinaria y tierra en el cultivo del arroz de la región central de Colombia $(n=85)$

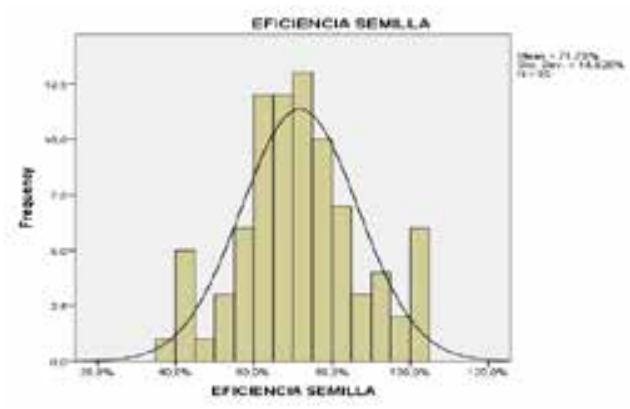


Revista LEBRET • No. 5 • Enero - Diciembre de 2013
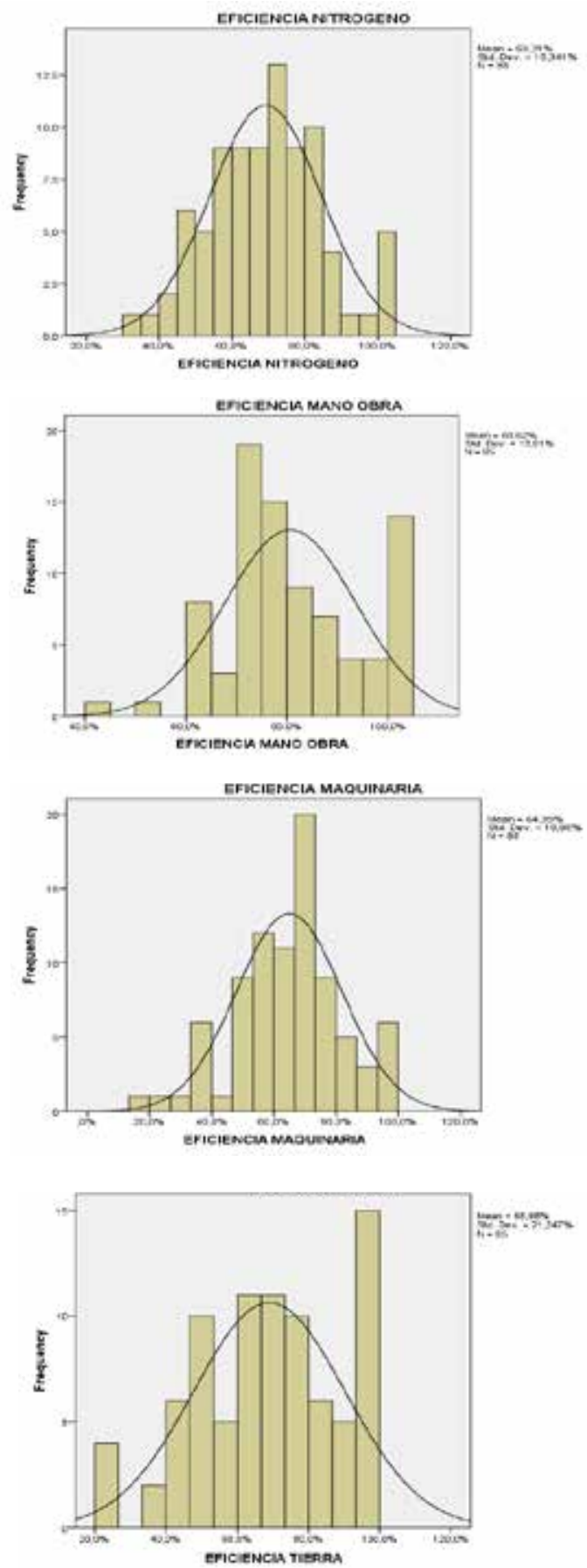

Fuente: Cálculos propios basados en datos de FEDEARROZ 
En general, se confirma que a nivel de los recursos individuales los resultados son consistentes con el índice promedio de eficiencia distributiva para todas las fincas incluidas en el análisis calculado en 64.6\% (Tabla 2) y, por tanto, existe un margen importante para reducir los costos unitarios de producción. Los índices variaron entre $65.0 \%$ para la maquinaria y $80.6 \%$ para la mano de obra sugiriendo que la maquinaria es el factor productivo usado menos eficientemente, seguido por el nitrógeno, tierra, y semilla.

Se arguye que las ineficiencias en maquinaria se deben principalmente a la obsolescencia del parque de tractores y combinadas cuya edad superaba más de 10 años en el 2007 (FEDEARROZ, 2008). Asimismo, se argumenta, a manera de hipótesis, que el uso de altas densidades de siembra (semillas) y la aplicación de altas dosis de nitrógeno contribuyen a explicar las ineficiencias distributivas encontradas. El uso de mayores cantidades de estos insumos parece constituir una prima de riesgo que los agricultores están dispuestos a pagar para contrarrestar el impacto en productividad por efecto de malezas, plagas y enfermedades y deficiencias en el manejo del agua de riego entre otras fuentes de pérdidas de rendimientos. En contraste, se aduce que el tamaño reducido de los cultivos y el predominio de cultivos operados en arriendo son fuentes importantes de ineficiencias distributivas. Por su parte, la creciente incorporación de mano de obra capacitada en el manejo de las labores del cultivo en los programas del SENA y de FEDEARROZ ayuda a entender la mayor eficiencia distributiva encontrada en la mano de obra.

Como se desprende del Tabla 3, los rangos de variación de la eficiencia distributiva entre fincas variaba ampliamente así: semilla (37.2 al 100\%); nitrógeno (33.9 al $100 \%$ ); mano de obra (44.9 al 100\%); maquinaria (18.5 al 100\%); y tierra (20.5 al $100 \%$ ). Por tanto, se confirma que los márgenes para reducir costos unitarios son amplios si los agricultores acceden a maquinaria y equipos modernos, reducen las cantidades actuales de semilla, aplican menores cantidades de nitrógeno y aumentan la eficiencia del nitrógeno sobre suelo seco, acceden a mayores cantidades de tierra de cultivo propia y de agua para riego, y vinculan personal capacitado a las labores. 
Tabla 3. Distribución del número de fincas arroceras según niveles de eficiencia distributiva en el uso de recursos productivos en la región central de Colombia

$$
(\mathrm{n}=85)
$$

\begin{tabular}{|c|c|c|c|c|c|c|c|c|c|c|}
\hline \multirow{2}{*}{$\begin{array}{l}\text { Rango del } \\
\text { índice de } \\
\text { Eficiencia } \\
\text { (\%) }\end{array}$} & \multicolumn{2}{|c|}{ Semilla } & \multicolumn{2}{|c|}{ Nitrógeno } & \multicolumn{2}{|c|}{ Mano de Obra } & \multicolumn{2}{|c|}{ Maquinaria } & \multicolumn{2}{|c|}{ Tierra } \\
\hline & $\begin{array}{l}\text { Número } \\
\text { fincas }\end{array}$ & $\%$ & $\begin{array}{l}\text { Número } \\
\text { fincas }\end{array}$ & $\%$ & $\begin{array}{l}\text { Número } \\
\text { fincas }\end{array}$ & $\%$ & $\begin{array}{l}\text { Número } \\
\text { fincas }\end{array}$ & $\%$ & $\begin{array}{c}\text { Número } \\
\text { fincas }\end{array}$ & $\%$ \\
\hline 3 a 13 & 0 & 0.0 & 0 & 0.0 & 0 & 0.0 & 0 & 0.0 & 0 & 0.0 \\
\hline 13 a 23 & 0 & 0.0 & 0 & 0.0 & 0 & 0.0 & 1 & 1.2 & 1 & 1.2 \\
\hline 23 a 33 & 0 & 0.0 & 0 & 0.0 & 0 & 0.0 & 1 & 1.2 & 3 & 3.5 \\
\hline 33 a 43 & 3 & 3.5 & 3 & 3.5 & 0 & 0.0 & 7 & 8.2 & 5 & 5.9 \\
\hline 43 a 53 & 6 & 7.1 & 11 & 12.9 & 2 & 2.4 & 10 & 11.8 & 13 & 15.3 \\
\hline 53 a 63 & 12 & 14.1 & 15 & 17.6 & 5 & 5.9 & 17 & 20.0 & 12 & 14.1 \\
\hline 63 a 73 & 24 & 28.2 & 23 & 27.1 & 19 & 22.4 & 25 & 29.4 & 15 & 17.6 \\
\hline 73 a 83 & 22 & 25.9 & 17 & 20.0 & 27 & 31.8 & 12 & 14.1 & 11 & 12.9 \\
\hline 83 a 93 & 9 & 10.6 & 10 & 11.8 & 12 & 14.1 & 6 & 7.1 & 10 & 11.8 \\
\hline 93 a 100 & 9 & 10.6 & 6 & 7.1 & 20 & 23.5 & 6 & 7.1 & 15 & 17.6 \\
\hline Promedio & $71.8 \mathrm{a}$ & & $69.3 \mathrm{a}$ & & $80.6 b$ & & $65.0 \mathrm{c}$ & & $69.0 \mathrm{a}$ & \\
\hline $\begin{array}{l}\text { Desviación } \\
\text { Estándar }\end{array}$ & 14.9 & & 15.3 & & 13.0 & & 17.0 & & 21.2 & \\
\hline Máximo & 100.0 & & 100.0 & & 100.0 & & 100.0 & & 100.0 & \\
\hline Mínimo & 37.2 & & 33.9 & & 44.1 & & 18.5 & & 20.8 & \\
\hline
\end{tabular}

Fuente: Cálculos propios. Las letras (a, b, c) indican que las diferencias en los promedios de eficiencia calculados son estadísticamente significativas con $\mathrm{P}<0.05$. Índices con la misma letra no son estadísticamente diferentes de acuerdo con una prueba t para comparación de medias apareadas.

\subsection{Eficiencia y tamaño del cultivo}

En la literatura reciente los estudios de eficiencia se caracterizan por estimar no sólo el nivel de eficiencia sino también los factores que explican las ineficiencias. El tamaño de operación ha sido identificado como una fuente principal de ineficiencias en el corto plazo, entre muchos otros factores socio-demográficos, agroecológicos y de dotación, acceso a recursos y servicios productivos por los agricultores. Sin embargo, los resultados no son consistentes, ya que en algunos estudios se han encontrado costos crecientes (retornos decrecientes) con la escala de operación y en otros, costos decrecientes (retornos crecientes) sugiriendo que la asignación de tierra no es la óptima. Este análisis es particularmente relevante en arroz, un cultivo donde predominan los microfundios y minifundios con tamaños de cultivo inferiores a 10 hectáreas. 
La Tabla 4 registra el valor y significancia del coeficiente de correlación parcial de Spearman entre los índices de eficiencia, y el tamaño de los cultivos de arroz. Los resultados confirman que, exceptuando la eficiencia económica total, hubo una asociación alta (correlación mayor a 0.500$)$ y significativa $(\mathrm{P}<0.01)$ entre el tamaño de los cultivos y los indicadores de eficiencia técnica y distributiva. Sin embargo, no se encontró asociación alguna con la eficiencia económica. Es de resaltar que la asociación con la eficiencia distributiva fue negativa. Esto se interpreta como que las fincas de mayor tamaño, son menos eficientes en usar los niveles y combinaciones de recursos para minimizar costos que las fincas más pequeñas. Este resultado es congruente con la teoría de Theodore Schultz, de que los agricultores que operan a pequeña escala son tradicionales y pobres pero eficientes.

Tabla 4. Coeficientes de correlación de Spearman entre índices de eficiencia y tamaño de los cultivos de arroz en la región central de Colombia $(n=85)$

\begin{tabular}{ccc}
\hline \multirow{2}{*}{ Eficiencia } & \multicolumn{2}{c}{ Tamaño Cultivo (ha) } \\
\cline { 2 - 3 } & $\begin{array}{c}\text { Coeficiente } \\
\text { correlación }\end{array}$ & Desviación estándar \\
\hline Técnica & $0.410^{* * *}$ & 0.097 \\
\hline Distributiva & $-0.858^{* * *}$ & 0.053 \\
\hline Económica & 0.02 & 0.982 \\
\hline Semilla & $0.775^{* * *}$ & 0.060 \\
\hline Nitrógeno & $0.756^{* * *}$ & 0.065 \\
\hline Mano de obra & $0.771^{* * *}$ & 0.058 \\
\hline Maquinaria & $0.678^{* * *}$ & 0.078 \\
\hline Tierra & $0.860^{* * *}$ & 0.036 \\
\hline
\end{tabular}

Fuente: Cálculos propios. $* * * \mathrm{P}<0,01$

La Tabla 5 muestra la distribución de los índices de eficiencia según las categorías de tamaño de predios aceptadas por el Instituto Geográfico Agustín Codazzi IGAC. Se puede observar que la eficiencia técnica aumenta con el tamaño del cultivo, mientras que la eficiencia distributiva se reduce con el incremento en el área dedicada al arroz. La eficiencia económica más alta se encontró en las fincas minifundistas con diferencias significativas con los restantes rangos de tamaño. En contraste, las fincas más grandes en la muestra (medianas) fueron las menos eficientes económicamente con un nivel equivalente al $50 \%$ de la eficiencia promedia de la muestra, no obstante registrar el mayor nivel de ingresos brutos por unidad de área sembrada y registrar los rendimientos más altos por hectárea.

Estos resultados ayudan a entender por qué no se encontró una correlación significativa entre eficiencia económica y tamaño del cultivo. También, soportan la tesis de Schultz de que los agricultores en microfundios y minifundios son eficientes 
distributivamente y maximizan las ganancias del cultivo usando los recursos en combinaciones de mínimo costo.

Tabla 5. Distribución de los índices de eficiencia en la producción de arroz en la región central de Colombia según categorías de tamaño de los cultivos $(n=85)$

\begin{tabular}{|c|c|c|c|c|c|c|}
\hline \multirow{2}{*}{$\begin{array}{l}\text { Categorías de } \\
\text { tamaño }\end{array}$} & \multirow{2}{*}{$\begin{array}{l}\mathrm{N} \\
\%\end{array}$} & \multicolumn{3}{|c|}{ Eficiencia } & \multirow{2}{*}{$\begin{array}{c}\text { Ingreso Bruto } \\
\text { \$ ha-1 }\end{array}$} & \multirow{2}{*}{$\begin{array}{c}\text { Rendimiento } \\
\text { kg ha-1 }\end{array}$} \\
\hline & & Técnica & Distributiva & Económica & & \\
\hline $\begin{array}{l}\text { Microfundios } \\
\quad(<3 \mathrm{ha})\end{array}$ & 31.8 & $\begin{array}{l}33,5 \mathrm{a} \\
(18,2)\end{array}$ & $\begin{array}{l}78,0 \mathrm{a} \\
(4,6)\end{array}$ & $\begin{array}{l}26,8 \mathrm{a} \\
(15,8)\end{array}$ & $\begin{array}{c}1.376 .515,5 \mathrm{a} \\
(549.362,4)\end{array}$ & $6.441,1 \mathrm{a} 1.014,1$ \\
\hline $\begin{array}{l}\text { Minifundio } \\
\text { (3 a } 12.9 \text { ha) }\end{array}$ & 52.9 & $\begin{array}{l}52,5 \mathrm{~b} \\
(21,4)\end{array}$ & $\begin{array}{l}62,7 b \\
(10,3)\end{array}$ & $\begin{array}{l}33,6 \mathrm{~b} \\
(14,9)\end{array}$ & $\begin{array}{c}1.574 .914,4 \mathrm{a} \\
(487.575,9)\end{array}$ & $\begin{array}{c}6.385,2 \mathrm{a} \\
(914,4)\end{array}$ \\
\hline $\begin{array}{c}\text { Pequeño } \\
\text { (13 a } 22.9 \text { ha) }\end{array}$ & 12.9 & $\begin{array}{l}53,1 \mathrm{~b} \\
(15,5)\end{array}$ & $\begin{array}{l}47,1 \mathrm{c} \\
(18,6)\end{array}$ & $\begin{array}{l}26,1 \mathrm{a} \\
(13,4)\end{array}$ & $\begin{array}{c}1.670 .071,1 \mathrm{a} \\
(452.145,0)\end{array}$ & $\begin{array}{c}6.439,8 \mathrm{a} \\
(778,7)\end{array}$ \\
\hline $\begin{array}{l}\text { Mediano } \\
(23 \text { ha }+)\end{array}$ & 2.4 & $\begin{array}{l}72,2 \mathrm{c} \\
(15,9)\end{array}$ & $\begin{array}{l}20,6 \mathrm{~d} \\
(17,4)\end{array}$ & $\begin{array}{l}16,3 \mathrm{c} \\
(15,8)\end{array}$ & $\begin{array}{c}2.211 .826,3 \mathrm{~b} \\
(186.380,5)\end{array}$ & $\begin{array}{c}7.051,0 \mathrm{~b} \\
(69,3)\end{array}$ \\
\hline Total & 100 & $\begin{array}{l}47,0 \mathrm{~d} \\
(21,7)\end{array}$ & $\begin{array}{l}64,6 \mathrm{e} \\
(15,9)\end{array}$ & $\begin{array}{l}30,1 \mathrm{f} \\
(15,3)\end{array}$ & $\begin{array}{c}1.539 .194,2 \mathrm{a} \\
(514.680,1)\end{array}$ & $\begin{array}{c}6.425,7 \mathrm{a} \\
(915,9)\end{array}$ \\
\hline
\end{tabular}

Fuente: Cálculos propios. Las letras (a, b, c) a lo largo de las columnas indican que las diferencias

en los promedios de eficiencia calculados son estadísticamente significativas con $\mathrm{P}<0.05$ de acuerdo con una prueba $\mathrm{t}$ para comparación de medias apareadas. Índices con la misma letra no son estadísticamente diferentes. Las letras (d, e, f) a lo largo de la fila total indican que las diferencias en los promedios de eficiencia calculados son estadísticamente significativas con $\mathrm{P}<0.05$. Valores entre paréntesis son rangos y desviaciones estándar.

\section{Conclusiones e implicaciones del estudio}

Este análisis sustenta la hipótesis central del estudio de que en la región central de Colombia, una zona relativamente homogénea en términos agroecológicos y tecnológicos para la producción de arroz riego, un cultivador promedio puede aumentar significativamente los rendimientos por hectárea (53\%), reducir los costos unitarios (34.5\%) y aumentar las ganancias brutas antes de cubrir los costos fijos (70\%) con la dotación actual de recursos y la tecnología disponible. Para ello, se necesita que los agricultores con ineficiencias económicas ajusten los niveles de uso y combinaciones de recursos a los empleados por los agricultores más eficientes en la muestra analizada. Estos últimos agricultores presentaron los rendimientos por hectárea más altos, los costos unitarios más bajos, y por tanto, las mayores ganancias por unidad de área.

De acuerdo con estos resultados se considera que para incrementar la productividady competitividad de la producción de arroz en la región central, en el corto plazo y de una manera efectiva en costos, se debería asignar prioridad 
y concentrar los recursos y esfuerzos en el fortalecimiento de los programas de transferencia de tecnología y capacitación de los agricultores, trabajadores y operarios y técnicos con el fin de entrenarlos y motivarlos hacia el uso más eficiente de los recursos productivos y de la tecnología existente teniendo como referente los materiales genéticos, el manejo agronómico y el desempeño técnico y económico del cultivo por los agricultores más eficientes en rendimientos y costos unitarios.

Mientras el estudio no identifica en forma explícita las fuentes de las ineficiencias encontradas, los datos analizados evidencian de que en función de aumentar la eficiencia técnica y distributiva, en el cultivo del arroz se presentan rendimientos crecientes o costos decrecientes al tamaño del cultivo en el uso de la tecnología. Por tanto, los agricultores que operan microfundios y minifundios con cultivos inferiores a 3 hectáreas, podrían aumentar apreciablemente su eficiencia técnica y económica actual si pueden acceder a mas recursos de tierra de cultivo a fin de hacer mayores economías de escala. Entre tanto, las fincas pequeñas y medianas (con cultivos mayores a 3 has) requieren ahorrar y combinar mejor el uso de semilla, nitrógeno, maquinaria y mano de obra a fin de aumentar su eficiencia técnica, distributiva y económica.

En este contexto, las instituciones del sector deberían ejecutar acciones de política de tierras tendientes a aumentar el tamaño actual de los cultivos de arroz en esta región con énfasis en los cultivadoresque operan unidades menores de 3 has a fin de aumentar su eficiencia en la escala de operación, mejorando su productividad y competitividad actual en la producción de arroz. Asimismo, a continuar fortaleciendo los esfuerzos de transferencia de tecnología y de capacitación hacia todos los tipos de productores pero con énfasis especial en los más pequeños que son la gran mayoría (84.7\% de los productores en la muestra).

\section{Referencias}

Admassie, A. (1999). Sources of efficiency differentials in smallholder agriculture. Quartely Journal of International Agriculture 38 (3), 203-220.

Ahmad, M., \& Bravo-Ureta, B. (1996). Technical efficiency measures for dairy farms using panel data: a comparison of alternative model specifications. The Journal of Productivity Analysis 7, 399-415.

Aigner, D. J., \& Chu, S. F. (1968). On estimating the industry production function. The American Economic Review 58, 826-839.

Aigner, D. J., Lovell, K. C., \& Schmidt, P. (1977). Formulation and estimation of stochastic production functions. Journal of Econometrics , 21-37. 
Ajibefun, I. A., Batesse, G. E., \& Daramola, A. G. (2002). Determinants of technical efficiency in smalholder food crop farming: an application of stochastic production functions. Quaterly Journal of International Agriculture, 225-240.

Ali, M., \& Flinn, J. C. (1989). Profit Efficiency among basmati rice producers in pakistan punjab. American Journal of Agricultural Economics 71 (2), 303-310.

Álvarez, A. (2001). La Medición de eficiencia y la productividad. Madrid: Editorial Pirámide.

Audibert, M. (1997). Technical Inefficiency Effects among Rice Paddy Farmers in Mali, West Africa. Journal of Productivity Analysis 8 (4), 379-394.

Batesse, G. E., \& Coelli, T. J. (1988). Prediction of Firm Level Technical Efficiencies with a Generalized Production Function and Panel Data. Journal of Econometrics, 387-399.

Batesse, G. E., \& Coelli, T. J. (1992). Frontier Production Functions, Technical Efficiency and Panel Data with Application to Rice Paddy Farms in India. Journal of Productivity Analysis 3 (1-2), 153-169.

Binam, J. T. (2004). Factors affecting the technical efficiency among smallholder farmers in the slash and burn agriculture zone of Cameroon. Food Policy 29 (5), $531-545$

Bravo-Ureta, B. E., \& Rieger, L. (1990). Alternative production frontier methodologies and dairy farm efficiency. Journal of Agricultural Economics 41, 215-226.

Bravo-Ureta, B. E., Solis, D., Moreira, U. H., Maxipani, J. F., Thiam, M., \& Rivas, T. (2007). Technical Efficiency in Farming: a Meta Regression Analysis. Journal of Productivity Analysis 27 (1), 57-72.

Brummer, B. (2001). Estimating confidence intervals for technical efficiency: the case of private farms in Slovenia. European review of Agricultural Economics 28 (3), 285-306.

Coelli, T. (1996). A Guide to frontier version 4.1: a computer program for frontier production function estimation. Armidale, Australia: Department of Econometrics, University of New England. CEPA Working Paper 96/07.

Coelli, T., \& Batesse, G. (1996). Identification of factors which influence the technical efficiency of indian farmers. Australian Journal of Agricultural and Resource Economics 40 (2), 103-128.

Coelli, T., Prasada, D. S., \& Batesse, D. S. (1998). An Introduction to efficiency and productivity analysis. Boston, Dordrecht and London: Kluwer Academics Publishers. 
Cooper, W. W., Seiford, L. M., \& Tone, K. (2007). Data Envelopment Analysis. New York, NY: Springer Science \& Business Media.

DANE, \& FEDEARROZ. (2011). Encuesta Nacional de Arroz Mecanizado. Bogota: FEDEARROZ.

Dawson, P. J. (1985). Measuring Technical Efficiency from Production Functions: Some Further Estimates. Journal of Agricultural Economics 36, 31-40.

Dawson, P. J. (1989). Measuring Farm Efficiency Over Time on Philippine Rice Farms. Journal of Agricultural Economics 40 , 168-177.

Dawson, P. J., \& Lingard, J. (1991). Approaches to Measuring Technical Efficiency on Philippine Rice Farms. Journal of International Development 3 (3), 211-228.

Dawson, P. J., Lingard, J., \& Woodford, C. H. (1991). A Generalized Measure of Farm Specific Technical Inefficiency. American Journal of Agricultural Economics, 1098-1104.

Dhungona, B. R., Nuthall, P. L., \& Nartea , G. V. (2004). Measuring the Economic Inefficiency of Nepalese Rice Farms using Data Envelopment Analysis. Australian journal of Agricultural and Resource Economics 48 (2), 347-369.

Dillon, J. L. (1968). The Analysis of Response in Crop and Livestock Production. Sidney, Australia: Pergamon Press Ltd.

Ekanayake, S. A., \& Jayasuriya, S. (1987). Measurement of Firm - Specific Technical Efficiency: A Comparison of Methods . Journal of Agricultural Economics 38 , $115-123$.

Farrell, M. J. (1957). The Measurement of Productive Efficiency. Journal Of the Royal Statistics Society 120(III), 259-275.

FEDEARROZ.(2012).Adopción Masivade Tecnología. Bogotá:FEDEARROZ:Fondo Nacional del Arroz.

FEDEARROZ, Federación Nacional de Arroceros;. (2008). III Censo Nacional Arrocero 2007. Bogotá: FEDEARROZ, División de investigaciones Económicas.

FEDESARROLlO, IQUARTIL, \& MADR. (2012). Costos de Producción de 12 Productos Agropecuarios. Bogotá: FEDESARROLLO.

Flinn, A. C., \& Ali, M. (1986). Technical Efficiency in Basmati Rice Production. Pakistan Journal of Applied Economics 5, 63-79.

Fried, H. O., Lovell, C. A., \& Schmidt, S. S. (1993). The Measurement of Productive Efficiency. New York: Oxford University Press. 
Galawat, F., \& Yabe, M. (2012). Evaluation of technical, allocative and economic efficiency in rice production: a case study of rice farmers in brunei Durusalam. Journal of the Faculty of Agriculture Kyushu University 57 (1), 317-325.

Gee-Thean, L., Ismail, M. M., \& Harron, M. (2012). Measuring technical efficiency of malasyan paddy farming: an application of stochastic production frontier approach. Journal of Applied Sciences 12 (15), 1602-1607.

Greene, H. W. (1993). Frontier production functions. New York: Working Paper EC93-20, Stern School of Business NYU.

Greene, W. H. (2002a). LIMDEP Version 8.0. New York: Pearson Education.

Greene, W. H. (2002b). Econometric Analysis. New York: Pearson Education.

Greene, W. H. (1980). Maximum likelohood estimation of econometric frontier functions. Journal of Econometrics (13), 27-56.

Heady, E. O. (1952). The Economics of agricultural production and resource use. Ames: Iowa: Iowa State University Press.

Heady, E. O., \& Dillon, J. L. (1961). Agricultural production functions. Ames, IO: Iowa State University Press.

Idiong, I. C. (2007). Estimation of farm level technical efficiency in small scale swamp rice production in cross river state of nigeria: a stochastic frontier approach. World Journal of Agricultural Sciences 3 (5), 653-658.

Islam, K. M., Sumelius , J., \& Backman, S. (2012). Do differences in technical efficiency explain the adoption rate of hyv rice? evidence from Bangladesh. Agricultural Economics Review (13 (1), 93-110.

Jaforullah, M., \& Premachandra, E. (2003). Sensitivity of technical efficiency estimates to estimation approaches: an investigation using new zealand dairy industry data. University of Otago Ecoomics Discusion Papers 0306, 1-23.

Kalijaran, K., \& Flinn, J. C. (1983). The Measurement of farm-specific technical efficiency. Pakistan Journal of Applied Economics 2, 167-181.

Kwon , S. O., \& Lee, H. (2004). Productivity Improvement in korean rice farming: parametric and non-parametric analysis. Journal of Agricultural and Resource Economics 48 (2), 323-346.

Lingard, J., Castillo, L., \& Jayasuriya, S. (1983). Comparative Efficiency of rice farms in central luzon, the philippines. Journal of Agricultural Economics 34, 163-173.

Liu, J. S., Lu, L. Y., Lu, W. M., \& Lin, B. Y. (2013). A Survey of DEA Applications. Omega (United Kingdom) 41 (1), 3-15. 
Liu, Z., \& Zhuang, J. (2000). Determinants of technical efficiency in post collective chinese agriculture: evidence from farm-level data. Journal of Comparative Economics 28 (3), 545-564.

Meeusen, W., \& Van Den Broeck, J. (1977). Efficiency estimation for cobb douglas production functions. International Economic Review 18, 435-443.

Minh, N. K., \& Long, G. T. (2009). Efficiency estimates for the agricultural production in vietnam: a comparison of parametric and non-parametric approaches. Agricultural Economics Review 10 (2), 62-78.

Neff, D. L., Garcia , P., \& Nelson, C. H. (1993). Technical efficiency: a comparison of production frontier methods. European Review of Agricultural Economics 21, 479-489.

Nguyen, T. M., Kawaguchi, T., \& Suzuki, N. (2003). A study on technical efficiency of rice production in the mekong delta-vietnam by stochastic frontier analysis. Journal ogf the Faculty of Agriculture, Kyushu university 48 (1-2), 325-357.

Nyemeck, B. J., Sylla, K., Diarra, I., \& Nyambi, G. (2003). Factors affecting technical efficiency among coffee farmers in cote $\mathrm{d}^{\prime}$ ivory: evidence from the centre west region. African Development Review 15 (1), 66-76.

Ortner, K. M., Hambrusch, J., \& Kirner, L. (2006). The Efficiency of dairy farms in austria: do natural conditions matter? Vienna: Federal Institute of Agricultural Economics.

Ramírez, A. (1986). Resource use and economic efficiency of rice production in colombia. Stillwater, OK: Oklahoma State University Ph. D. Dissertation.

Ramirez, A., \& Sanint, L. R. (1994). Eficiencia económica de factores de producción y prioridades para el manejo de tecnología en arroz riego: una aplicación de funciones de producción frontera. Cali: Programa de Arroz CIAT.

Richmond, J. (1974). Estimating the efficiency of production. International Economic Review 15, 515-521.

Sharma, K. R., Leung, P., \& Zalesqui, H. M. (1999). Technical, allocative and economic efficiencies in swine production in hawai: a comparison of parametric and non-parametric approaches. Agricultural Economics 20, 23-35.

Shehu, J. F., Mshelia, S. I., \& Tashikalma, A. K. (2007). Analysis of techical efficiency of small-scale rainfed upland rice farmers in northwest Nigeria. Journal of Agriculture and Social Sciences 3 (4), 133-136.

Simar, L., \& Wilson, P. (2000). A General Methodology for Bootstrapping in NonParametric Frontier Models. Journal of Applied Statistics 27 (6), 779-802. 
Tadesse, B., \& Krishnamoorty, S. A. (1997). Technical efficiency in paddy farms of Tamil Nadu: an analysis based on farm size and ecological zone. Agricultural Economics 16 (3), 185-192.

Thiam, A., Bravo-Ureta, B. E., \& Rivas, T. E. (2001). Technical efficiency in developing country agriculture: a meta-analysis. Agricultural Economics 25, 235 243.

Tian, W., \& Wan , G. H. (2000). Technical efficiency and its determinants in China's Grain Production. Journal of Productivity Analysis, 159-174.

Trewin, R., Weiguo, L., \& Erwidodo, B. S. (1995). Analysis of the technical efficiency over time of west javanese rice farms. Australian Journal of Agricultural Economics 39 (2), 143-163.

Wadud, A. (2003). Technical, Allocative, and economic efficiency of farms in Bangladesh: a Stochastic Frontier and DEA Approach. The Journal of Developing Areas 37 (1), 109-126.

Wadud, A., \& White, B. (2000). Farm Household efficiency in Bangladesh: a comparison of stochastic frontier and DEA methods. Applied Economics 32 (13), 1665-1673.

Xu, X., \& Jeffrey, S. R. (1998). Efficiency and technical progress in rice production in China traditional and modern agriculture. Agricultural Economics 18 (2), 157165. 